\title{
El derecho de defensa en el proceso inmediato por flagrancia delictiva en el Primer Juzgado de Investigación preparatoria de San Martín - Tarapoto, 2018
}

\author{
The right of defense in the immediate process for criminal \\ flagrance in the First Preparatory Investigation court of San \\ Martín - Tarapoto, 2018 \\ Yamunaqué Gonzáles, Jessica Paola. ${ }^{1[0000-0002-4203-161 X]}$ \& Moreno \\ Aguilar, Jhin Demetrio 1 [0000-0003-4958-9605]. \\ ${ }^{1}$ Universidad Nacional de San Martín, Tarapoto, Perú \\ jdmoreno@unsm. edu.pe
}

Citar como: Yamunaqué Gonzáles, J. P. \& Moreno Aguilar, J. D. (2021). El derecho de defensa en el proceso inmediato por flagrancia delictiva en el Primer Juzgado de Investigación preparatoria de San Martín - Tarapoto, 2018. Revista Científica Ratio Iure, 1(2), 49-58. https://doi.org/10.51252/rc ri.v1i2.197

Recibido: 19/04/2021

Aceptado: 20/06/2021

Publicado: 28/07/2021
Resumen. El presente artículo titulado "El derecho de defensa en el proceso inmediato por flagrancia delictiva en el Primer Juzgado de Investigación Preparatoria de San Martín-Tarapoto, año 2018"; tiene como objetivo principal determinar de qué manera se vulnera el derecho de defensa en el proceso inmediato por flagrancia delictiva en el Primer Juzgado de Investigación Preparatoria de San Martín-Tarapoto, 2018. La investigación es de tipo básico, a nivel descriptivo, de un diseño no experimental, donde la muestra de estudio estuvo conformada por 30 expedientes judiciales, los instrumentos que se utilizaron para recoger información fue la guía de observación y encuesta realizada a los operadores del derecho entre jueces, fiscales y abogados de la ciudad de Tarapoto. Finalmente, de los 30 expedientes y de las 30 encuestas realizadas se llegaron a la conclusión que se vulnera el derecho de defensa en el proceso inmediato por flagrancia delictiva debido a que el plazo establecido es corto para que la defensa técnica del imputado pueda obtener y presentar las pruebas que acrediten su teoría de caso, debido a que aún falta regular nuevos plazos del Decreto Legislativo $\mathrm{N}^{\circ} 1194$.

Palabras clave: Delito flagrante, derecho de defensa, flagrancia delictiva, proceso inmediato, procedimiento penal.

Abstract. This article entitled "The right of defense in the immediate process due to criminal flagrancy in the First Preparatory Investigation Court of San Martín-Tarapoto, year 2018"; Its main objective is to determine how the right of defense is violated in the immediate process due to criminal flagrancy in the First Preparatory Investigation Court of San Martín-Tarapoto, 2018; and as specific objectives to analyze the establishment of the immediate process by criminal flagrance and to analyze the right of defense in the immediate process by criminal flagrance. The investigation is of a basic type, at a descriptive level, of a non-experimental design, where the study sample was made up of 30 judicial files, the instruments used to collect information were the document analysis guide and the survey of the operators of law among judges, prosecutors and lawyers of the city of Tarapoto. Finally, the results show that, from the 30 files and the 30 surveys carried out, it was concluded that the right of defense is violated in the immediate process due to criminal flagrance because the established deadline is short for the technical defense of the accused can obtain and present the evidence that proves his case theory, because there is still a need to regulate new terms of Legislative Decree $\mathrm{N}^{\circ} .1194$.

Keywords: Criminal flagrance, criminal procedure, defense law, flagrant crime, immediate process. 


\section{$1 \quad$ Introducción}

El 30 de agosto del dos mil quince se dictó el Decreto Legislativo $\mathrm{N}^{\circ} 1194$, el cual regula el Proceso Inmediato, mediante la precitada norma, se modificaron los artículos 446, 447 y 448 del Nuevo Código Procesal Penal (Decreto Legislativo No 957), donde señala como los aspectos más relevantes: a) Se impone su aplicación en supuestos de flagrancia, confesión o acumulación de evidentes elementos de convicción, y b) Se impone expresamente en ilícitos como los de Omisión de Asistencia Familiar y Conducción en Estado de Ebriedad o Drogadicción.

Con este decreto, se establece obligatoriedad para que el fiscal incoe proceso inmediato, con la finalidad de eliminar la delincuencia, así como, lograr la celeridad de los procesos penales; dándole potestad al fiscal de interponer acusación cuando éste cuenta con todos los medios probatorios suficientes para imputarle responsabilidad al detenido, y por ello resulta indispensable, que tal actividad sea llevada a cabo con responsabilidad, y evitando la vulneración de derechos fundamentales como el derecho a la defensa.

El proceso inmediato se calcula que dura entre 4 días a 7 días, lo que se busca en esta investigación es indicar que no es posible que se prefiera la celeridad procesal en sacrificio del derecho de defensa, un claro ejemplo de ello es el caso de Buscaglia Zapler, la cual fue condenada por desobediencia a la autoridad por un total de 6 años con 8 meses, con la motivación de una sentencia basada en un video que no muestra la totalidad de los hechos e incumpliendo el principio de proporcionalidad de la pena.

Teniéndose en consideración que una vez pasada la audiencia de incoación el fiscal tiene un día para presentar su acusación, observando claramente que no es aceptable que el fiscal con la premura del tiempo pueda recolectar las pruebas suficientes que acrediten la responsabilidad del imputado y a su vez, que ocurre con la defensa, pues se deja prácticamente sin tiempo alguno para analizar y realizar nuevas actuaciones para la audiencia de juzgamiento en dos días posteriores, imposibilitándole tener un tiempo prudente para revisar el expediente y presentar nuevas pruebas que acrediten la inocencia del inculpado.

Se debe comprender que la efectividad de una norma no solamente implica que se castigue severamente o erradique a la delincuencia, sino que la norma debe basarse en un procedimiento garantista y respetuoso de los derechos y principios generales de un proceso penal acusatorio respetuoso de lo que dispone su constitución, por lo tanto el legislador al momento de emitir la norma debió contemplar estos supuestos y no emitir una modificatoria que disminuye garantías procesales constitucionales; pues esta institución jurídica privilegia la celeridad a costo de sacrificar derechos fundamentales. 
Bajo estos enunciados se busca establecer la importancia y necesidad de resguardar el respeto irrestricto al derecho de defensa en el proceso inmediato por flagrancia delictiva., pues, la aplicación del Decreto Legislativo $\mathrm{N}^{\circ} 1194$, trae consigo la vulneración al derecho de defensa, al plazo razonable y a la prueba, ya que debido al escaso tiempo estipulado para la duración de este proceso, acarrea significativas dificultades, al momento de obtener los elementos de convicción suficientes que sustenten la adecuada defensa del imputado, originando que las decisiones jurisdiccionales puedan ser arbitrarias o desproporcionales.

\section{Materiales y Métodos}

\subsection{Tipo de investigación}

El tipo de investigación es básica, para Landeau, (2007), se fundamenta en un argumento teórico y su intención fundamental consiste en desarrollar una teoría, extender, corregir o verificar el conocimiento mediante el descubrimiento de amplias divulgaciones o principios. Este tipo de investigación se realiza para obtener nuevos conocimientos y nuevos campos de investigación sin un fin práctico específico e inmediato. Tiene como fin crear un cuerpo de conocimiento teórico, sin preocuparse de su aplicación práctica. Se orienta a conocer y persigue la resolución de problemas amplios y de validez general.

\subsection{Nivel de investigación}

El nivel de investigación es descriptiva, según Hernández Sampieri et al., (2014): los estudios descriptivos buscan especificar propiedades y características importantes de cualquier fenómeno que se analice. Describe tendencias de un grupo o población. Es decir, únicamente pretenden medir o recoger información de manera independiente o conjunta sobre los conceptos o las variables a las que se refieren.

\subsection{Diseño}

Diseño no experimental: la investigación es de diseño no experimental; porque en el presente trabajo no se manipula las variables deliberadamente, sino que se observaron los casos ocurridos en el medio natural; "su propósito es describir variables y analizar su incidencia e interrelación en un momento dado" Hernández Sampieri et al., (2014).

Donde:

El diseño es: 


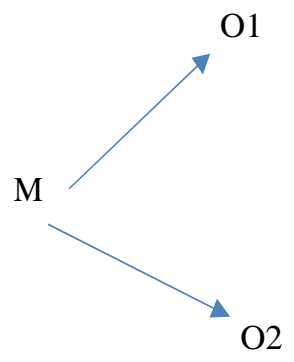

Dónde:

M: Representa la muestra de estudio conformado por los expedientes judiciales físicos.

O1: Derecho de defensa.

O2: Proceso inmediato por flagrancia delictiva.

\subsection{Población y muestra}

\section{Población.}

La población está compuesta por 70 expedientes judiciales de proceso inmediato por flagrancia delictiva.

\section{Muestra.}

La presente investigación aplica el tipo de muestreo no probabilístico, para el registro de datos, debido al carácter de la información que se necesita recabar.

Dentro del tipo de muestreo no probabilístico, para seleccionar a la muestra se acudirá al método de muestreo por conveniencia, por el cual se seleccionará directa e intencionadamente a los individuos de la población.

Conformado por 30 expedientes judiciales de proceso inmediato por flagrancia delictiva.

\subsection{Materiales}

Los materiales documentales usados son:

- Jurisprudencias

- Doctrina

- Libros jurídicos

- Leyes

- Decretos Legislativos 


\subsection{Métodos}

Para Solís Espinoza, (2008) explica: "método sirve de instrumento para alcanzar los fines de la investigación; su carácter regular, explicito, perceptible, ordenado y objeto para lograr algo, estable que la investigación ha de seguir para alcanzar un fin".

En si el método es aquel medio con el cual el investigador realizó un procedimiento lógico cognitivo, en cada etapa de la tesis, con el fin de lograr una respuesta conectada con los objetivos de estudio. Sustentando así los supuestos de investigación.

Con respecto a los métodos utilizados en la investigación, tenemos los siguientes conceptos:

Método argumentativo: Es el método que busca fundamentos con apoyo de la lógica y la razón para sustentar alguna afirmación.

Método inductivo: Es aquel que busca sacar una conclusión general de la distinta información recopilada de temas puramente específicos.

Método deductivo: Es aquel método que tiene la finalidad de concluir en un tema específico de toda la información general recopilada.

\section{$3 \quad$ Resultados y discusiones}

Tabla 1

Realiza acciones la defensa técnica para ejercer correctamente el derecho de defensa

\begin{tabular}{|lllllc}
\hline & & Frecuencia & Porcentaje & $\begin{array}{l}\text { Porcentaje } \\
\text { Válido }\end{array}$ & $\begin{array}{c}\text { Porcentaje } \\
\text { acumulado }\end{array}$ \\
\hline \multirow{3}{*}{ Válido } & $\mathrm{Si}$ & 11 & 36,7 & 36,7 & 36,7 \\
\cline { 2 - 6 } & No & 19 & 63,3 & 63,3 & 100,0 \\
\cline { 2 - 6 } & Total & 30 & 100,0 & 100,0 & \\
\hline
\end{tabular}

En la Tabla 1, se observa que el 63,3\% (19) de la muestra manifiesta que no realiza acciones la defensa técnica para ejercer correctamente el derecho de defensa, y solo un 36,7 \% (11) si realiza acciones la defensa técnica.

Tabla 2

Aporta pruebas la parte de la defensa técnica del imputado

\begin{tabular}{llllll}
\hline & & Frecuencia & Porcentaje & $\begin{array}{l}\text { Porcentaje } \\
\text { válido }\end{array}$ & $\begin{array}{l}\text { Porcentaje } \\
\text { Acumulado }\end{array}$ \\
\hline \multirow{3}{*}{ Válido } & $\mathrm{Si}$ & 6 & 20,0 & 20,0 & 36,7 \\
& No & 24 & 80,0 & 80,0 & 100,0 \\
\cline { 2 - 6 } & No & 30 & 100,0 & 100,0 & \\
\hline
\end{tabular}

En la Tabla 2, se observa que el $80 \%$ (24) de la muestra manifiesta que no aporta pruebas la parte de la defensa técnica del imputado, y un $20 \%$ (6) si aporta pruebas la defensa técnica. 
Tabla 3

Cuenta con pruebas suficientes el abogado defensor del imputado para acreditar su teoría del caso

\begin{tabular}{clllcc}
\hline & & Frecuencia & Porcentaje & $\begin{array}{l}\text { Porcentaje } \\
\text { válido }\end{array}$ & $\begin{array}{c}\text { Porcentaje } \\
\text { acumulado }\end{array}$ \\
\hline \multirow{3}{*}{ Válido } & $\mathrm{Si}$ & 8 & 26,7 & 26,7 & 26,7 \\
& $\mathrm{No}$ & 22 & 73,3 & 73,3 & 100,0 \\
\cline { 2 - 6 } & Total & 30 & 100,0 & 100,0 & \\
\hline
\end{tabular}

En la Tabla 3, se observa que el 73,3\% (22) de la muestra manifiesta que no cuenta con pruebas suficientes el abogado defensor del imputado para acreditar su teoría del caso, y solo un 26,7 \% (8) si cuenta con pruebas suficientes el abogado defensor del imputado.

Tabla 4

Perjudica la excesiva celeridad al plazo razonable para que el abogado pueda obtener pruebas de descargo.

\begin{tabular}{c|lllc|c}
\hline \multirow{2}{*}{ Válido } & Frecuencia & Porcentaje & $\begin{array}{c}\text { Porcentaje } \\
\text { válido }\end{array}$ & $\begin{array}{c}\text { Porcentaje } \\
\text { acumulado }\end{array}$ \\
\hline \multirow{3}{*}{} & $\mathrm{Si}$ & 18 & 60,0 & 60,0 & 60,0 \\
& $\mathrm{No}$ & 12 & 40,0 & 40,0 & 100,0 \\
\cline { 2 - 6 } & Total & 30 & 100,0 & 100,0 & \\
\hline
\end{tabular}

En la Tabla 4, se observa que el 60,0\% (18) de la muestra manifiesta que si perjudica la excesiva celeridad al plazo razonable para que el abogado pueda obtener pruebas de descargo, y un 40,0 $\%$ (12) no perjudica la excesiva celeridad al plazo razonable.

Tabla 5

¿Cree Usted que la regulación del proceso inmediato por flagrancia delictiva vulnera el derecho de defensa, el plazo razonable y al derecho a presentar pruebas?

\begin{tabular}{ccccccccc}
\hline Encuestados & \multicolumn{2}{c}{ Jueces } & \multicolumn{2}{c}{ Fiscales } & \multicolumn{2}{c}{ Abogados } & \multicolumn{2}{c}{ Total } \\
\hline Respuestas & $\mathrm{N}^{\circ}$ & $\%$ & $\mathrm{~N}^{\circ}$ & $\%$ & $\mathrm{~N}^{\circ}$ & $\%$ & $\mathrm{~N}^{\circ}$ & $\%$ \\
\hline Si & 3 & $10 \%$ & 6 & $20 \%$ & 9 & $30 \%$ & 18 & $60 \%$ \\
No & 7 & $24 \%$ & 4 & $13 \%$ & 1 & $3 \%$ & 12 & $40 \%$ \\
\hline
\end{tabular}

En la Tabla 5, se observa que el 10\% de Jueces, 20\% de Fiscales y 30\% de Abogados manifiestan que, la regulación del proceso inmediato por flagrancia delictiva SI vulnera el derecho de defensa, el plazo razonable y el derecho a presentar; y un 24\% de Jueces, $13 \%$ de Fiscales y $3 \%$ de Abogados manifiestan NO se vulnera el derecho de defensa, ni el plazo razonable, ni el derecho a presentar pruebas.

Observando en la figura $\mathrm{N}^{\circ} 07$ el total de las repuestas, donde el $60 \%$ de los encuestados entre Jueces, Fiscales y Abogados afirman que SI se vulnera y el $40 \%$ que NO.

Tabla 6

¿Es suficiente el plazo de 72 horas que da el proceso inmediato por flagrancia delictiva para la designación de abogado defensor y construir la estrategia de defensa?

\begin{tabular}{ccccccccc}
\hline Encuestados & \multicolumn{2}{c}{ Jueces } & \multicolumn{2}{c}{ Fiscales } & \multicolumn{2}{c}{ Abogados } & \multicolumn{2}{c}{ Total } \\
\hline Respuestas & $N^{\circ}$ & $\%$ & $N^{\circ}$ & $\%$ & $N^{\circ}$ & $\%$ & $N^{\circ}$ & $\%$ \\
\hline Si & 6 & $20 \%$ & 3 & $10 \%$ & 2 & $7 \%$ & 11 & $37 \%$ \\
No & 4 & $13 \%$ & 7 & $23 \%$ & 8 & $27 \%$ & 19 & $63 \%$ \\
\hline
\end{tabular}


En la Tabla 6, se observa que el 20\% de Jueces, $10 \%$ de Fiscales y $7 \%$ de Abogados manifiestan que, SI es suficiente el plazo de 72 horas que da el proceso inmediato por flagrancia delictiva para la designación de abogado defensor y construir la estrategia de defensa; y un 13\% de Jueces, 23 $\%$ de Fiscales y $27 \%$ de Abogados manifiestan que NO es suficiente el plazo.

Tabla 7

¿Considera razonable la realización y actuación de pruebas para la acusación en el plazo de 2 a 4 días desde la detención?

\begin{tabular}{ccccccccc}
\hline Encuestados & \multicolumn{2}{c}{ Jueces } & \multicolumn{2}{c}{ Fiscales } & \multicolumn{2}{c}{ Abogados } & \multicolumn{2}{c}{ Total } \\
\hline Respuestas & $\mathrm{N}^{\circ}$ & $\%$ & $\mathrm{~N}^{\circ}$ & $\%$ & $\mathrm{~N}^{\circ}$ & $\%$ & $\mathrm{~N}^{\circ}$ & $\%$ \\
\hline Si & 3 & $10 \%$ & 5 & $17 \%$ & 2 & $7 \%$ & 10 & $33 \%$ \\
No & 7 & $23 \%$ & 5 & $17 \%$ & 8 & $27 \%$ & 20 & $67 \%$ \\
\hline
\end{tabular}

En la Tabla 7, se observa que el 10\% de Jueces, 17\% de Fiscales y 7\% de Abogados manifiestan que, SI es razonable la realización y actuación de pruebas para la acusación en el plazo de 2 a 4 días desde la detención; y un 23\% de Jueces, 17 \% de Fiscales y 27\% de Abogados manifiestan que NO es razonable la realización y actuación de pruebas.

\section{Tabla 8}

En su experiencia, ¿considera usted que el hecho que los acusados se acogen a la terminación anticipada del proceso, es por el poco tiempo que tienen para presentar pruebas que les permita un juicio en igualdad de armas?

\begin{tabular}{ccccccccc}
\hline Encuestados & \multicolumn{2}{c}{ Jueces } & \multicolumn{2}{c}{ Fiscales } & \multicolumn{2}{c}{ Abogados } & \multicolumn{2}{c}{ Total } \\
\hline Respuestas & $\mathrm{N}^{\circ}$ & $\%$ & $\mathrm{~N}^{\circ}$ & $\%$ & $\mathrm{~N}^{\circ}$ & $\%$ & $\mathrm{~N}^{\circ}$ & $\%$ \\
\hline Si & 8 & $27 \%$ & 6 & $20 \%$ & 7 & $23 \%$ & 21 & $70 \%$ \\
No & 2 & $7 \%$ & 4 & $13 \%$ & 3 & $10 \%$ & 9 & $30 \%$ \\
\hline
\end{tabular}

En la Tabla 8, se observa que el 27\% de Jueces, 20\% de Fiscales y 23\% de Abogados manifiestan que, los acusados SI se acogen a la terminación anticipada del proceso por el poco tiempo que tienen para presentar pruebas que les permita un juicio en igualdad de armas; y un 7\% de Jueces, $13 \%$ de Fiscales y $10 \%$ de Abogados manifiestan que NO se acogen a la terminación anticipada.

Tabla 9

¿Se respeta la libertad probatoria del imputado, en el proceso inmediato por flagrancia delictiva?

\begin{tabular}{ccccccccc}
\hline Encuestados & \multicolumn{2}{c}{ Jueces } & \multicolumn{2}{c}{ Fiscales } & \multicolumn{2}{c}{ Abogados } & \multicolumn{2}{c}{ Total } \\
\hline Respuestas & $\mathrm{N}^{\circ}$ & $\%$ & $\mathrm{~N}^{\circ}$ & $\%$ & $\mathrm{~N}^{\circ}$ & $\%$ & $\mathrm{~N}^{\circ}$ & $\%$ \\
\hline $\mathrm{Si}$ & 3 & $10 \%$ & 5 & $17 \%$ & 2 & $7 \%$ & 10 & $33 \%$ \\
No & 7 & $23 \%$ & 5 & $17 \%$ & 8 & $27 \%$ & 20 & $67 \%$ \\
\hline
\end{tabular}

En la Tabla 9, se observa que el 10\% de Jueces, 17\% de Fiscales y 7\% de Abogados manifiestan que, SI se respeta la libertad probatoria del imputado, en el proceso inmediato por flagrancia delictiva; y un 23\% de Jueces, $17 \%$ de Fiscales y $27 \%$ de Abogados manifiestan que NO se respeta la libertad probatoria. 


\section{Tabla 10}

¿En el corto plazo que se da en el proceso inmediato por flagrancia delictiva, los imputados se encuentran restringidos a presentar medios de prueba?

\begin{tabular}{ccccccccc}
\hline Encuestados & \multicolumn{2}{c}{ Jueces } & \multicolumn{2}{c}{ Fiscales } & \multicolumn{2}{c}{ Abogados } & \multicolumn{2}{c}{ Total } \\
\hline Respuestas & $\mathrm{N}^{\circ}$ & $\%$ & $\mathrm{~N}^{\circ}$ & $\%$ & $\mathrm{~N}^{\circ}$ & $\%$ & $\mathrm{~N}^{\circ}$ & $\%$ \\
\hline $\mathrm{Si}$ & 6 & $20 \%$ & 8 & $27 \%$ & 8 & $27 \%$ & 22 & $73 \%$ \\
$\mathrm{No}$ & 4 & $13 \%$ & 2 & $7 \%$ & 2 & $7 \%$ & 8 & $27 \%$ \\
\hline
\end{tabular}

En la Tabla 10, se observa que el 20\% de Jueces, 27\% de Fiscales y 27\% de Abogados manifiestan que, en el corto plazo que se da en el proceso inmediato por flagrancia delictiva, los imputados SI se encuentran restringidos a presentar medios de prueba; y un 13\% de Jueces, $7 \%$ de Fiscales y $7 \%$ de Abogados manifiestan que NO se encuentran restringidos a presentar medios de pruebas.

Observando la tabla 10 el total de las repuestas, donde el $73 \%$ de los encuestados entre Jueces, Fiscales y Abogados afirman que en el corto plazo que se da en el proceso inmediato por flagrancia delictiva, los imputados SI se encuentran restringidos a presentar medios de prueba y el $27 \%$ que NO se encuentran restringidos.

\section{Tabla 11}

¿En el corto plazo que se da en el proceso inmediato por flagrancia delictiva, cuenta el abogado defensor del imputado con prueba suficiente para acreditar su teoría del caso?

\begin{tabular}{ccccccccc}
\hline Encuestados & \multicolumn{2}{c}{ Jueces } & \multicolumn{2}{c}{ Fiscales } & \multicolumn{2}{c}{ Abogados } & \multicolumn{2}{c}{ Total } \\
\hline Respuestas & $\mathrm{N}^{\circ}$ & $\%$ & $\mathrm{~N}^{\circ}$ & $\%$ & $\mathrm{~N}^{\circ}$ & $\%$ & $\mathrm{~N}^{\circ}$ & $\%$ \\
\hline $\mathrm{Si}$ & 2 & $7 \%$ & 1 & $3 \%$ & 0 & $0 \%$ & 3 & $10 \%$ \\
$\mathrm{No}$ & 8 & $27 \%$ & 9 & $30 \%$ & 10 & $33 \%$ & 27 & $90 \%$ \\
\hline
\end{tabular}

En la Tabla 11, se observa que el 7\% de Jueces, 3\% de Fiscales y 0\% de Abogados manifiestan que, en el corto plazo que se da en el proceso inmediato por flagrancia delictiva, SI cuenta el abogado defensor del imputado con prueba suficiente para acreditar su teoría del caso; y un 27\% de Jueces, $30 \%$ de Fiscales y 33\% de Abogados manifiestan que NO cuentan con prueba suficiente.

\section{Conclusiones}

El Decreto Legislativo $\mathrm{N}^{\circ}$ 1194-Proceso inmediato fue creado con la finalidad de ser un proceso célere para combatir con la delincuencia; sin embargo, desde su modificación hasta la actualidad viene generando vulneraciones al derecho de defensa.

El derecho a la defensa está expresamente reconocido en nuestra Constitución Política, por lo que todas las propuestas legislativas en materia procesal penal se sujetan al principio de jerarquía normativa. Por lo que no cabe aceptar la reducción de la garantía constitucional del derecho defensa en el proceso inmediato por flagrancia, por mínima que esta sea; toda vez que no deben ser estos derechos sacrificados en aras de la celeridad procesal. 
El proceso inmediato por flagrancia delictiva vulnera el derecho de defensa, pues el imputado al contar con plazos tan breves le es imposible jurídica y materialmente intervenir en el proceso bajo los principios de contradicción e igualdad de armas, a su vez le impide recabar y ofrecer pruebas, afectando así al derecho a probar y al núcleo constitucional del derecho a la defensa.

Es urgente cambiar los plazos establecidos en el proceso inmediato por flagrancia, ya que fue previsto para durar un plazo no mayor a seis días calendarios, la misma que es inaceptable porque no permite realizar una defensa efectiva; y este cambio evitaría la vulneración de derechos constitucionales del imputado, así como al derecho de defensa, al derecho a probar y al plazo razonable, pues la importancia de entregar un tiempo razonable para que la defensa técnica realice la estrategia de defensa es fundamental e inherente para la imparcialidad del proceso.

\section{Referencias bibliográficas}

Araya, V. A. (2016). Nuevo Proceso Inmediato para Delitos en Flagrancia. Gaceta jurídica. Carrasco, M. A. (2016). La implicancia Del Proceso Inmediato Por Flagrancia Delictiva Al Principio Acusatorio y Al Derecho A Ser Juzgado en un plazo razonable. [Universidad de Huanuco.].

http://repositorio.udh.edu.pe/handle/123456789/173; jsessionid=22A6D77A72D618E8928 B531BFBF8AB47

Cartagena, H. Y. (2016). La aplicación del proceso inmediato a raíz de la modificatoria del Decreto Legislatvo $N^{\circ} 1194$ colisiona con el Derecho de Defensa, en los Juzgados Penales de la Provincia de Sicuani: Un analisis a partir de la experiencia. [Universidad Andina del Cusco]. http://repositorio.uandina.edu.pe/handle/UAC/473

Cubas, V. V. (2006). El proceso penal.(Sexta). Palestra Editores.

Evans, D. 1. (1986). Los Derechos Constitucionales. Editorial Juridica de Chile.

Hernandez, S. R. (2014). Metodología de la Investigación. (6). Interamericana Editores S.A. Landeau, R. (2017). Elaboración de trabajos de investigación. (1 $\left.{ }^{\mathrm{a}}\right)$. Editorial Alfa Venezuela. Mendoza Ayma, F. C. (2016). El control de la detención en flagrancia y el Proceso Inmediato. Revista Ius in fraganti, 1(1), 44-47.

Monge, H. V. (2012). La constitucionalidad del proceso penal de flagrancia. [Universidad de Costa Rica].

Nakasaki, S. C. (2017). El derecho Penal y Procesal Penal desde la perspectiva del abogado penalista litigante. Gaceta Jurídica.

Reategui, S. J. (2016). El proceso penal inmediato en casos de flagrancia delictiva. Ediciones Legales.

Reyes Catalán, A. (2004). El delito Flagrante: Sus implicancias en el Proceso Penal.

[Universidad Austral de Chile]. 
http://cybertesis.uach.cl/tesis/uach/2004/fjr456d/pdf/fjr456d.pdf

Rosas, Y. J. (2014). Los sujetos procesales en el nuevo Código Procesal Penal. Lex \& luris.

Ruiz Fernádez, S. (2017). La implicancia de la cuasiflagrancia y el plazo razonable por parte de la defensa en el proceso inmediato, Lima Norte. [Universidad Cesar Vallejo]. https://repositorio.ucv.edu.pe/handle/20.500.12692/23309

Saldaña Terrones, C. S. (2016). Los efectos jurídicos sobre los principios y derechos del marco penal en el proceso inmediato en delitos de flagrancia Lima. [Universidad Cesar Vallejo]. https://repositorio.ucv.edu.pe/handle/20.500.12692/1603

San Martín Castro, C. (1999). Derecho Procesal Penal. Grijley.

Solis Espinoza, A. (2018). Metodología de la Investigación Jurídico Social. [Lima: Perú].

Trujillo Giraldo, J. R. (2017). Vulneracion del Derecho a la Defensa en los casos de flagrancia aplicados al proceso inmediato en el Distrito Fiscal de Ventanilla en el año 2016 Huánuco. [Universidad Nacional Hermilio Valdizar]. http://repositorio.unheval.edu.pe/handle/UNHEVAL/3871

\section{Conflicto de intereses}

Los autores indican que no existen conflictos de interés.

\section{Contribuciones de los autores}

La contribución de cada autor se ha realizado de manera coordinada en todo el proceso de la investigación y elaboración del artículo científico.

Copyright (c) 2021 Jessica Paola Yamunaqué Gonzáles \& Jhin Demetrio Moreno Aguilar

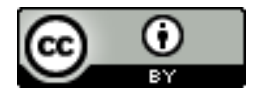

Este texto está protegido por una licencia CreativeCommons 4.0.

\footnotetext{
Usted es libre para Compartir — copiar y redistribuir el material en cualquier medio o formato- y Adaptar el documento — remezclar, transformar y crear a partir del material— para cualquier propósito, incluso para fines comerciales, siempre que cumpla la condición de:

Atribución: Usted debe dar crédito a la obra original de manera adecuada, proporcionar un enlace a la licencia, e indicar si se han realizado cambios. Puede hacerlo en cualquier forma razonable, pero no de forma tal que sugiera que tiene el apoyo del licenciante o lo recibe por el uso que hace de la obra.

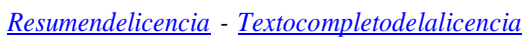

